\title{
Programa Farmácia Popular do Brasil: uma análise política de sua origem, seus desdobramentos e inflexões
}

\author{
Popular Pharmacy Program of Brazil: a political analysis of its origin, \\ unfoldings and inflections
}

\author{
Tatiane de Oliveira Silva Alencar ${ }^{\mathbf{1}}$, Patrícia Sodré Araújo², Ediná Alves Costa², Rafael Damasceno \\ Barros4, Yara Oyram Ramos Lima5, Jairnilson Silva Paim 6
}

1 Universidade Estadual de Feira de Santana (UEFS)

- Feira de Santana (BA),

Brasil.

Orcid: https://orcid.

org/0000-0001-6257-

5633

tatifarmauefs@yahoo.com.br

2 Universidade do

Estado da Bahia (Uneb),

Departamento de Ciências

da Vida - Salvador (BA),

Brasil.

Orcid: https://orcid.

org/0000-0001-5843-

5662

patrisodre@gmail.com

3 Universidade Federal da Bahia (UFBA), Instituto de Saúde Coletiva (ISC) Salvador (BA), Brasil.

Orcid: https://orcid. org/0000-0002-2195-

3180

eacosta98@gmail.com

4 Universidade Federal da Bahia (UFBA), Escola de Enfermagem - Salvador (BA), Brasil.

Orcid: https://orcid

org/000-0002-1782-7253

rafaeldbarros@hotmail.com

5 Universidade Federal da Bahia (UFBA), Instituto de Saúde Coletiva (ISC) Salvador (BA), Brasil. Orcid: https://orcid. org/0000-0002-22521566

yaraoyram@yahoo.com.br

${ }^{6}$ Universidade Federal da Bahia (UFBA), Instituto de Saúde Coletiva (ISC) -

Salvador (BA), Brasil.

Orcid: https://orcid

org/0000-0003-0783-

$262 X$

jairnil@ufba.br
DOI: $10.1590 / 0103-110420185211$

RESUMO O artigo se refere a uma investigação qualitativa que analisa as origens, os desdobramentos, resultados e desafios do Programa Farmácia Popular do Brasil, face à necessária integralidade da assistência farmacêutica. Para a produção dos dados, foram utilizados documentos e entrevistas semiestruturadas com informantes envolvidos com o movimento da Reforma Sanitária Brasileira e/ou a questão medicamentos/assistência farmacêutica, considerando o período de 2003 a 2016; notícias produzidas entre 2013 e 2018, disponíveis em sítios eletrônicos de instituições entendidas como atores no processo de implementação de políticas farmacêuticas. A análise dos dados revelou que, apesar dos conflitos em torno da construção da proposta, o programa resultou em ampliação do acesso aos medicamentos essenciais, redução da mortalidade e de internações por doenças crônicas. Por esse motivo, inflexões no programa, como o fechamento da modalidade rede própria, resultaram em posicionamentos de organizações da sociedade civil, também motivados diante da conjuntura de perdas de direitos sociais. Permanecem lacunas concernentes à qualidade do acesso em suas diversas dimensões de análise e à promoção do uso racional de medicamentos, na perspectiva da integralidade da Assistência Farmacêutica.

PALAVRAS-CHAVE Políticas públicas. Controle de medicamentos e entorpecentes. Assistência Farmacêutica. Preparações farmacêuticas.

ABSTRACT The article refers to a qualitative research that analyzes the origins, unfoldings, results and challenges of the Brazilian Popular Pharmacy Program, in view of the necessary integrality of pharmaceutical assistance. For the production of the data, documents and semistructured interviews were used with informants involved with the Brazilian Health Reform movement and the issue of medicines/pharmaceutical assistance, considering the period from 2003 to 2016; news produced between 2013 to 2018, available on electronic sites of institutions understood as actors in the process of implementing pharmaceutical policies. Analysis of the data revealed that, despite the conflicts surrounding the proposal's construction, the program resulted in increased access to essential drugs, reduced mortality and hospitalizations for chronic diseases. For this reason, inflections in the program, such as the closing of the first modality, 
have resulted in positions of civil society organizations, also motivated by the conjuncture of losses of social rights. Gaps remain regarding the quality of access in its various dimensions of analysis and the promotion of rational use of medicines, from the perspective of the integrality of Pharmaceutical Assistance.

KEYWORD Public policy. Drug and narcotic control. Pharmaceutical services. Pharmaceutical preparations.

\section{Introdução}

O debate sobre acesso a medicamentos constou da agenda da saúde antes mesmo da instituição do Sistema Único de Saúde (SUS); foi ampliado e incorporou novas dimensões, à medida que foram implementadas políticas de saúde visando à integralidade da atenção e ao direito à Assistência Farmacêutica $(\mathrm{AF})^{\mathbf{1}, \mathbf{2}}$. Os termos Medicamentos e 'Assistência Farmacêutica' apresentam concepções distintas, técnica e politicamente, no processo de constituição das políticas farmacêuticas nos diferentes momentos históricos ${ }^{3}$.

Nesse processo, o direito universal a medicamentos fundamentou um conjunto de políticas, cujo marco foi a Política Nacional de Medicamentos (PNM), em 1998, e a Política Nacional de Assistência Farmacêutica (PNAF), em 2004, que definiram diretrizes e ações norteadoras para outras políticas ao longo dos 30 anos do SUS.

A despeito dessas políticas, persistem lacunas em termos de regulação sanitária, uso racional de medicamentos, serviços farmacêuticos clínicos, farmacovigilância, articulação entre as políticas instituídas e disponibilidade de medicamentos ${ }^{4-7}$. O último aspecto motivou a formulação de políticas para promover a ampliação do acesso, a exemplo do Programa Farmácia Popular do Brasil (PFPB), criado em 2004, inicialmente, na modalidade rede própria e, depois, em convênio com farmácias privadas, inaugurando o sistema de copagamento no País.
No decorrer dos catorze anos do PFPB, houve conquistas na ampliação do acesso ${ }^{\mathbf{8}, 9}$, redução da mortalidade e de internações por doenças crônicas10; e também críticas, por suas características, estruturação, elenco, custos, desarticulação com a política industrial, relações com o setor farmacêutico privado etc."1. Seguiram-se inflexões, intensificadas na conjuntura regressiva, denominada austeridade fiscal, que tem marcado o país nos últimos anos.

O PFPB constitui uma das políticas farmacêuticas mais discutidas recentemente e também tem sido objeto de debates do Observatório de Análise Política em Saúde (OAPS), que, por meio de eixos temáticos, tem acompanhado essas políticas, especialmente nos componentes medicamentos, assistência farmacêutica e vigilância sanitária, tendo como referente o processo da Reforma Sanitária Brasileira (RSB). Considerando os questionamentos e as incertezas sobre o programa, e, na tentativa de fornecer elementos para ampliar o debate, o objetivo deste artigo é analisar o PFPB, suas origens, desdobramentos, resultados e desafios à necessária integralidade da AF.

\section{Estratégia metodológica}

Trata-se de uma investigação qualitativa, do tipo estudo de caso, resultado de análises das relações da RSB com a questão Medicamentos/Assistência Farmacêutica 
(questão MAF) ${ }^{\mathbf{1 2}}$, considerando o recorte temporal 2003 a 2016, e do acompanhamento das políticas farmacêuticas no eixo temático 'Políticas de Medicamentos, Assistência Farmacêutica e Vigilância Sanitária', do OAPS [Projeto Análise de Políticas de Saúde no Brasil (2003-2017) - Chamada MCTI/ CNPq/CT-Saúde/MS/SCTIE/Decit n ${ }^{\circ}$ 41/2013], no período 2013 a 2018.

Para a produção dos dados, foram utilizados dois conjuntos de fontes, que possibilitaram a construção do seguinte plano de análise:

a) Documentos e entrevistas semiestruturadas com informantes envolvidos com o movimento da RSB e/ou a questão MAF. As entrevistas foram guiadas por um roteiro, e os informantes consentiram na divulgação dos seus nomes. O estudo foi aprovado pelo CEP-ISC/UFBA, sob $\mathrm{n}^{\circ}$ 30623514.9.0000.5030. No processamento do material empírico, utilizou-se a técnica de análise de conteúdo temática ${ }^{13}$, e a análise dos dados foi orientada pelos seguintes elementos teóricos: contribuições da teoria marxista para entender o medicamento como um produto do ciclo produtivo-econômico da saúde e como uma necessidade social e existencial ${ }^{14,15}$; e as definições de poder, política, estratégia e ator social, segundo Testa ${ }^{16,17}$.

b) Fontes do OAPS, no eixo 'Políticas de Medicamentos, Assistência Farmacêutica e Vigilância Sanitária'. A produção dos dados baseou-se na seleção de informações, prioritariamente no link notícias, disponíveis em sítios eletrônicos de instituições entendidas como atores no processo de implementação de tais políticas: Ministério da Saúde (MS), Agência Nacional de Vigilância Sanitária (Anvisa), Conselho Nacional de Saúde (CNS), Conselho Nacional de Secretários de Saúde, Conselho Nacional de Secretarias Municipais de Saúde, Centro Brasileiro de Estudos de Saúde (Cebes) e Associação Brasileira de Saúde Coletiva (Abrasco). Também foram consultados sítios dos conselhos profissionais de Farmácia e Medicina
(CFF e CFM), a revista Radis-Fiocruz e a produção do Legislativo e do Executivo Federais. Organizou-se o material em planilhas padronizadas e individualizadas para cada sítio de consulta, atendo-se à seleção de fatos que apresentavam possibilidades de virem a se constituir em um fato político. Nesse caso, tomou-se como referencial a discussão sobre fatos sociais, históricos e políticos produzida pelo OAPS1,2,12.

\section{Origens e controvérsias do PFPB}

A primeira iniciativa no âmbito do Legislativo sobre um programa de farmácias populares foi o Projeto de Lei (PL) no 4.702/1994, de autoria do deputado Eduardo Jorge (Partido dos Trabalhadores - PT-SP), justificado pela dificuldade de acesso a medicamentos pela população face aos altos preços. Foi inspirado nas experiências de farmácias populares do Laboratório Farmacêutico do Estado de Pernambuco ${ }^{12}$ e no Programa Farmácia de Todos, do Rio Grande do Norte ${ }^{\mathbf{1 8}}$. Farmácias populares foram definidas no PL como estabelecimentos farmacêuticos de caráter privado que, por meio de franquia com o Estado, comercializariam medicamentos a preços reduzidos, tabelados, com margem de lucro definida.

Enquanto tramitava na Câmara dos Deputados, o PL foi identificado pela equipe do candidato Luiz Inácio Lula da Silva (Lula), no período de eleições à presidência de 2002 , como interessante à sua proposta de campanha, dada a dimensão simbólica e o valor de publicidade que poderia agregar. Assim, a ideia das farmácias populares foi inserida na campanha do candidato, e, com sua vitória, essa pauta tornou-se prioridade do MS, sem debate coletivo para a estruturação da proposta.

Durante a XII Conferência Nacional de Saúde, o ministro da saúde, Humberto Costa, anunciou a instalação de farmácias populares no País, sem detalhamento 
das características do programa. Naquele momento, surgiam polêmicas, e, durante as conferências de saúde municipais, ocorreram manifestações contrárias ao projeto. Argumentava-se que as unidades de saúde do SUS deveriam ampliar a disponibilidade de medicamentos para garantir acesso a todos, sem custo adicional, e que os laboratórios oficiais não dariam conta de atender a essa demanda adicional ${ }^{12}$.

A primeira discussão sobre o tema no âmbito do CNS ocorreu, simultaneamente, à apresentação da PNAF. O programa foi apresentado pelo então coordenador do Departamento de Assistência Farmacêutica (DAF-MS), explicando que tal medida relacionava-se com a PNAF e integraria um conjunto de ações do programa do governo Lula visando a ampliar o acesso a medicamentos, com a colaboração técnica e administrativa da Fundação Oswaldo Cruz (Fiocruz). Para seu financiamento, haveria alocação de recursos, por crédito suplementar, e assim não seria utilizado o orçamento da saúde ${ }^{12}$. Essas características geraram questionamentos por parte dos oponentes do programa, omitidos ou amenizados naquele momento, no CNS, até porque não seria estratégico mostrar possíveis confrontos, justamente quando havia relação de forças propícia ao desenvolvimento de ações relativas à questão $\mathrm{MAF}^{3}$.

Apesar da insistência do presidente Lula em implantar tal proposta, não houve consenso na equipe executora do MS nem entre os trabalhadores da Fiocruz, que teriam responsabilidade na execução do programa, pois realizariam as ações inerentes a produção ou aquisição, estocagem, comércio e dispensação de medicamentos:

Não foi uma definição técnica, não houve uma definição de nenhum de nós que participou da campanha. [...] E eu lhe digo, com toda a franqueza, tanto eu quanto toda minha equipe éramos contra criar um programa tipo o Farmácia Popular [...]. No entanto, nos deparamos com a seguinte questão: o tempo técnico é diferente do tempo político. O tempo técnico [relacionado às mudanças pretendidas para a assistência farmacêutica] por vez é muito maior do que o tempo político [no que se refere às contradições e conflitos no plano dos serviços]. [...] Ou seja, a gente pode não ter o tempo técnico necessário. (Norberto Rech).

Não era pra a Fiocruz cumprir isso. Nós não tínhamos nada a ver com isso [...]. A Fiocruz assumir a coordenação do [Programa] Farmácia Popular é algo que foge totalmente a seus objetivos como instituição de ciência e tecnologia de apoio ao SUS. [... ] engolimos esse sapo! Não só engolimos o Farmácia Popular como a coordenação do programa. (Álvaro Nascimento).

Ainda que houvesse um movimento contrário, as entidades farmacêuticas, como o CFF e a Federação Nacional de Farmacêuticos (Fenafar), expressaram apoio face à possibilidade de ampliação do mercado de trabalho farmacêutico e de práticas em ambientes mais adequados que os estabelecimentos privados $^{\mathbf{1 2}}$. Essas entidades vislumbravam a inserção dos farmacêuticos nas farmácias populares como possibilidade de avançar na implementação dos serviços farmacêuticos na atenção básica e articular outras demandas de entidades farmacêuticas.

O PFPB foi instituído pelo Decreto $\mathrm{n}^{\circ}$ $5.090 / 2004$, que regulamentou a Lei $\mathrm{n}^{\circ}$ 10.858/2004, e dispôs que a criação do programa decorria da necessidade de ações para promover a universalidade do acesso aos medicamentos, diminuindo a repercussão desses gastos no orçamento familiar e permitindo a sua disponibilidade a baixo custo para os cidadãos assistidos pela rede privada.

Parece ter havido preocupações em esclarecer a desvinculação do PFPB da disponibilização de medicamentos pelo SUS, provavelmente em função das críticas de inconstitucionalidade do programa ao fornecer medicamentos em copagamento, com prejuízos para as farmácias públicas ${ }^{19}$. O 
decreto esclarecia que o PFPB seria executado sem prejuízos para o abastecimento da rede pública.

\section{Desdobramentos: expansão da modalidade Aqui tem Farmácia Popular}

Dois anos após sua criação, o PFPB foi ampliado para farmácias da rede privada, conforme previa o Decreto $n^{0} 5.090 / 2004$, e os cidadãos puderam adquirir medicamentos para o tratamento de hipertensão e diabetes mediante copagamento. O MS repassaria às farmácias $90 \%$ do valor de referência do produto, conforme valores definidos pela Câmara de Regulação de Medicamentos, e o cliente pagaria $10 \%$. Fixaram-se critérios para credenciamento de farmácias ao PFPB, dispensação de medicamentos, controle e fiscalização, processamento de compras e comunicação ao público pelas farmácias ${ }^{\mathbf{1 2}}$.

A estratégia de expansão, Aqui Tem Farmácia Popular (ATFP), guardava mais semelhanças com o projeto original do deputado Eduardo Jorge, com as proposições iniciais do CFF e políticas farmacêuticas de outros países, com sistemas de saúde universais que dispunham de diferentes parâmetros e modalidades de copagamento ${ }^{\mathbf{1 8}, 20,21}$.

A modalidade ATFP gerou muitas críticas sob o argumento de que o Estado estimularia o mercado farmacêutico em vez de reforçar as farmácias do SUS, alvo de constantes queixas pela indisponibilidade de medicamentos. Posicionamentos divergentes também foram identificados entre militantes do movimento sanitário:

Então, quando se pensou no ATFP, a primeira pergunta era: tem sentido manter uma estrutura pública de dispensação paralela [modalidade rede própria]? Minha resposta: não, porque é muito mais caro, o sistema é corrupto [...], muito desperdício, problemas graves de estocagem
[...]. Então, a ideia foi romper com isso. [...]. Então, começamos com o ATFP [...]. (José Gomes Temporão).

Então, como é que se consegue fazer uma rede de dispensação viável? Utilizando a rede de dispensação privada, que já existe, que é o que faz a Itália, Portugal, Inglaterra, França, Alemanha [...]. A primeira proposta, que era essa da rede própria, se ela der certo, nada mais dará errado. (Gonzalo Vecina Neto).

Eu não sabia que a coisa podia piorar ainda mais! Em termos de proposta pode-se até dizer: 'Que legal vender remédio com desconto na farmácia comercial. Bacana'. Mas isso [...] é o que chamo de rebaixamento da pauta. [...] de certa maneira, uma parte dos sanitaristas está aceitando essa pauta rebaixada, reduzida, focalizada, fragmentada, que melhora, mas não resolve. (Suely Rozenfeld).

As parcerias com as farmácias privadas ampliariam o acesso a medicamentos, sobretudo para usuários com hipertensão e diabetes $\mathbf{1}$, principal meta do programa, além do argumento de um dos entrevistados, de ser um modelo mais econômico. Contudo, deve-se relativizar a comparação feita pelos entrevistados com o modelo de outros países (Itália, Portugal, Inglaterra, França, Alemanha), que possuem sistemas de saúde universais, pois há grandes diferenças entre os modelos de organização dos serviços e das práticas farmacêuticas naqueles países e no Brasil22.

Nos referidos países, as farmácias estão articuladas aos serviços de saúde, os serviços farmacêuticos clínicos integram os serviços disponíveis à população, e o farmacêutico tem função social e sanitária consolidada ${ }^{23}$. Isso não ocorre no Brasil, onde as farmácias privadas descumprem disposições legais e técnicas e funcionam, em muitos casos, sem farmacêutico, além de serem percebidas pela população como estabelecimentos meramente comerciais, pois não há prática de serviços clínicos ${ }^{12,24}$, algo incipiente em 
farmácias públicas. Amplo estudo realizado na atenção básica no SUS identificou que apenas $32,7 \%$ dos responsáveis pela entrega de medicamentos eram farmacêuticos5. Desses, apenas 21,3\% afirmaram realizar atividades de natureza clínica ${ }^{22}$.

Observe-se que se, naquele primeiro momento, o modelo de farmácia da rede própria do PFPB foi concebido para se contrapor ao das farmácias privadas, a modalidade ATFP se expandiu exatamente sob a lógica capitalista, que considera o medicamento como mercadoria, conquanto, em tese, a acreditação preservasse as exigências sanitárias legais e técnicas nas farmácias. Porém, muitos desses estabelecimentos não apresentavam, como não apresentam, estrutura capaz de realizar ações de promoção do uso racional dos medicamentos. Ou seja, o governo assumiu o modelo de farmácia criticado por entidades farmacêuticas e militantes da $\mathrm{RSB}^{12}$, e, ainda que promovesse a ampliação do acesso, a disponibilização de serviços farmacêuticos, defendida pelos sujeitos/atores sociais vinculados à questão MAF, permanecia como meta distante.

Entre consensos e contradições, o PFPB se ampliou nas duas modalidades. Até o fim do primeiro governo Lula, havia 259 farmácias da rede própria, distribuídas em 206 municípios, e 2.955 farmácias acreditadas no ATFP, em 594 municípios, com expansão em ambas do número de usuários ${ }^{7}$.

Ocorreu ampliação do acesso a medicamentos principalmente na modalidade rede própria. Entre 2005 e 2007 , houve expansão de atendimentos (cerca de 16 milhões), com potência de cobertura de 92 milhões de pessoas em 200718. Até 2010, houve crescimento expressivo do número de farmácias (de 27 para 543) e de municípios cobertos (de 7 para 420) 7 . Autores mostraram que os usuários de serviços de saúde privados foram aqueles que mais utilizaram o programa, com diferenças regionais, atendendo, portanto, à população alvo delimitada pelo programa ${ }^{7,18}$.
Tais dados expressavam a consolidação do programa, e, em 2011, houve reforço com a campanha 'Saúde Não Tem Preço', que passou a disponibilizar medicamentos para doenças específicas (hipertensão e diabetes não dependente de insulinas e, em seguida, para asma), todos isentos de copagamento nas farmácias da rede própria e naquelas conveniadas ao ATFP. Estratégia que teve rápida adesão da rede varejista9,12.

A Pesquisa Nacional de Saúde6 considerou o PFPB como uma política que contribui para o acesso a medicamentos. Das 19,3 milhões de pessoas que tiveram prescrição de medicamento no último atendimento de saúde, $21,9 \%$ conseguiram obter pelo menos um no referido programa, observando-se menor proporção no Nordeste ${ }^{6}$. Entre idosos, a prevalência de utilização foi de $57 \%$, e de conhecimento do programa, $87 \%{ }^{\mathbf{1 8}}$. Esses dados indicam o alcance do PFPB na articulação com as farmácias privadas, reduzindo barreiras de acesso geográfico e econômico.

Apesar da ampliação da cobertura e do acesso, persistiam dúvidas quanto à efetividade do programa para a expansão da AF em sua integralidade, conforme a PNAF. Ou seja, numa perspectiva para além da dimensão técnica do medicamento, que inclua a dimensão sociossanitária, com ações de natureza clínica, informação/educação para os usuários e promoção do uso racional, com potencialidade, portanto, para reverter a lógica de AF baseada numa concepção mais procedimental e centrada no medicamento, para outra centrada no cidadão e em suas necessidades ${ }^{25}$.

Conforme o MS4, após dez anos, o PFPB apresentava resultados positivos, sendo identificado como o programa social do governo federal mais bem avaliado pela população. Nesse período, atendeu mais de 32 milhões de pessoas e promoveu mais acesso a medicamentos para doenças crônicas (hipertensão, diabetes e asma), especialmente com o Saúde Não Tem Preço. O PFPB articulou-se a outros programas de governo, Plano Brasil sem Miséria, Brasil Carinhoso, Plano 
de Enfrentamento das Doenças Crônicas Não Transmissíveis etc., e recebeu reconhecimento internacional pela Organização Mundial de Saúde (OMS), como exemplo bem-sucedido para ampliação do acesso a medicamentos ${ }^{26}$.

Percebe-se que o PFPB se inseriu numa perspectiva de expansão do consumo, como outras políticas sociais do governo Lula para favorecer o desenvolvimento econômico. Ao criar condições para ampliação do consumo de medicamentos, ampliou-se a necessidade de produção, mantendo-se o ciclo produtivo econômico ${ }^{15}$ da saúde no País.

Pereira ${ }^{9}$ analisou a relação do PFPB com o Complexo Econômico-Industrial da Saúde, com dados do Ims Health, que demonstram que, em 2011, foram comercializadas 80,5 bilhões de doses de medicamentos no mercado varejista. Em 2012, observou-se crescimento de $11,8 \%$, somando 90 bilhões de doses. O PFPB participou com 6,0\% no volume de vendas do mercado varejista, em 2011, e com 7,7\%, em 2012.

Entretanto, para Scaramuzzo ${ }^{27}$, a principal ênfase do PFPB deve-se ao papel impulsionador do mercado de genéricos, exemplificado pelos resultados de janeiro de 2011, quando os genéricos correspondiam a $51 \%$ dos medicamentos comercializados nas farmácias do ATFP. Esse percentual passou para $56 \%$ em fevereiro daquele ano, após o lançamento do Saúde Não Tem Preço. Em agosto, a participação dos genéricos alcançou 65\%. Segundo a Associação Brasileira das Indústrias de Medicamentos Genéricos, no caso dos medicamentos para diabetes, o PFPB participou com $70 \%$ do total dos genéricos vendidos entre 2005 e $2011^{28}$.

O PFPB não foi criado como estratégia provisória, com período de duração definido, e nem mesmo a decisão de sua criação foi precedida de cálculo prévio sobre recursos, custos e demandas, como propõe o conceito de estratégia programática de Testa $^{16}$, pois foi uma decisão política e não técnica ${ }^{12}$. Restaria indagar sobre a efetividade do PFPB face à integralidade da $\mathrm{AF}$, pois essa concepção restrita de $\mathrm{AF}$ é a mesma que pode explicar as inflexões que ocorreram em seguida, sob o argumento de elevados custos necessários à manutenção da estrutura.

Um estudo ${ }^{11}$ criticou o PFPB ao comparar seu elenco de medicamentos com as listas nacional e internacional (Rename e Model List of Essencial Medicines), identificando incoerências: o elenco do PFPB apresentou medicamentos desnecessários quando cotejado às referidas listas; falta clareza nos critérios de inclusão e exclusão de medicamentos; constam formas farmacêuticas não registradas na Anvisa e outros medicamentos além daqueles do componente básico. Ademais, há falta de alinhamento do programa com a produção dos laboratórios oficiais.

Outros estudos ${ }^{7,29}$, incluindo resultados divulgados pelo MS após dez anos do $\mathrm{PFPB}^{4}$, nada referem sobre a qualidade dos serviços prestados. Isso demonstra que não se efetivou o pensamento inicial da categoria farmacêutica quanto à possibilidade de o PFPB produzir um novo modelo de farmácia, centrado na promoção do uso racional de medicamentos e na educação em saúde.

Estudos de avaliação econômica do programa revelaram maiores custos no ATFP em comparação com as farmácias públicas. Ao avaliar a diferença de custos entre a provisão de medicamentos por uma Secretaria Municipal de Saúde e pelo ATFP, Silva e Caetano 29 identificaram maiores custos no programa, indicando que alguns valores de referência poderiam ser examinados para sua redução, visando à sustentabilidade. Garcia e Acúrcio $^{30}$ realizaram um estudo de avaliação econômica dos programas da rede pública de farmácias do SUS, em Minas Gerais - Rede Farmácia de Minas Gerais -, comparados ao ATFP, e concluíram que: a produção direta dos serviços na rede pública mostrou-se economicamente mais favorável que a terceirização no ATFP; a vantagem econômica do setor público relaciona-se diretamente à sua capacidade de atendimento de pacientes; o investimento num modelo público integrado 
com princípios e diretrizes do SUS pode oferecer condições adequadas para garantir $\mathrm{AF}$ integral e universal de qualidade.

Ao cotejar o modelo de serviços farmacêuticos num mix público/privado, segmentado em farmácia pública estatal ou pública sob gestão de OSS coexistindo com o PFPB, Vieira ${ }^{31}$ sustenta que o acesso a medicamentos é facilitado na rede privada, contudo, ressalta possíveis dificuldades na gestão pública: sobreposição de programas com custos diferenciados para a mesma fonte pagadora e indução do mercado para garantir oferta apenas pelo programa que the seja mais rentável; os custos podem ser mais elevados que nos outros modelos; dificuldades de regulação e controle dos gastos; desarticulação entre os componentes técnico e logístico da AF; a fragmentação do cuidado pode ser maior, e o acompanhamento farmacoterapêutico pela gestão pública fica prejudicado, a menos que sejam criados mecanismos para interligar os serviços de saúde do SUS à rede privada ${ }^{31}$. Os custos podem ser maiores para o Estado no modelo segmentado, em que drogarias atuam na disponibilização de medicamentos e são ressarcidas pelo governo, tanto pelo produto quanto pelo serviço. O pensamento de Vieira ${ }^{31}$ corrobora os resultados de estudos que compararam os custos da oferta de medicamentos na $\mathrm{AF}$ pública aos do $\mathrm{PFPB}$, e concluíram que os custos são menores no SUS ${ }^{29,30}$.

\section{Inflexão na trajetória do PFPB e posicionamento de atores}

Apesar dos resultados positivos na ampliação do acesso a medicamentos, as polêmicas sobre o programa permaneceram, conquanto amenizadas. Em 2011, o ATFP foi auditado pelo Tribunal de Contas da União (TCU), que identificou: problemas no processo de acreditação de farmácias; falta de estudos que justificassem o custo-efetividade do programa como estratégia de complemento à $\mathrm{AF}$ na atenção básica; controles internos frágeis; inexistência de atividades para identificar condutas fraudulentas; e insuficiente apenação administrativa de irregularidades verificadas nos processos analisados ${ }^{\mathbf{1 2}}$.

O TCU solicitou esclarecimentos ao DAF/ MS e recomendou a elaboração de estudo para avaliar o custo-efetividade do programa, não identificado até o momento. Visando a reduzir os problemas, o MS publicou diversas portarias introduzindo mudanças e estabelecendo: cooperação com o Ministério da Previdência para acesso à base de dados do Sistema de Óbitos e cruzamento de dados do cadastro dos clientes do ATFP, evitando fraudes na aquisição de medicamentos; redução dos valores de referência dos medicamentos para diabetes e hipertensão; proibição do envio de medicamentos para as residências dos usuários; entrega gratuita de medicamentos; aumento das regras de identificação do paciente para dispensação de medicamentos ${ }^{3,12}$.

A partir de 2015, ocorreu inflexão no PFPB. Cortes orçamentários no governo Dilma impactaram o programa, que passou a assegurar gratuidade apenas dos medicamentos vinculados ao Saúde Não Tem Preço, asma, hipertensão e diabetes ${ }^{\mathbf{1}, 2}$. Novas medidas de controle, em 2016, abrangeram o credenciamento de farmácias privadas, que deixou de ser automático. Com o entendimento de que a inexistência de informações de âmbito nacional compromete diretamente a gestão da $\mathrm{AF}$ pelos entes federativos, o PFPB foi incluído na Base Nacional de Dados de Ações e Serviços da Assistência Farmacêutica no âmbito do SUS.

Em 2017, no contexto de retrocessos nas políticas sociais no governo Temer, diversas medidas provocaram significativas mudanças no Programa: introdução de critérios restritivos no acesso a medicamentos, critérios de idade para o fornecimento de medicamentos por patologias e redução do elenco. Em abril de 2017², a Comissão Intergestores Tripartite (CIT) decidiu pela interrupção do 
financiamento da modalidade rede própria, sob alegação de elevado custo administrativo. A estratégia anunciada pelo $\mathrm{MS}^{2}$ foi realocar os recursos, anteriormente destinados à rede própria do $\mathrm{PFPB}$, para a aquisição de medicamentos pelos estados e municípios na rede SUS, resultando no aumento do valor repassado pelo MS aos estados e municípios. Até agosto de $2017^{2}$, as unidades da rede própria foram extintas, restando a rede credenciada do ATFP, alvo de críticas e questionamentos pelo TCU, não esclarecidos pelo DAF/MS.

As alterações no PFPB têm sido entendidas por alguns atores, que se manifestaram publicamente, como retrocesso das políticas sociais produzidas no governo Lula, com repercussões no acesso aos medicamentos, especialmente para os mais pobres ${ }^{2}$.

O presidente do CNS criticou o fechamento da rede própria do PFPB, a Comissão Intergestores Tripartite (CIT) e o argumento utilizado pelo ministro da saúde, de que o custo administrativo dessa modalidade era pouco custo-efetivo e que o acesso aos medicamentos não seria afetado. Para o presidente do CNS, a extinção da rede própria e a redução da lista de medicamentos no ATFP dificultarão o acesso, gerando prejuízos à população².

A CIT foi questionada pela decisão unilateral em reunião apenas de gestores, sem representação de trabalhadores e usuários do SUS, ressaltando que não pode haver restrição do acesso da população ao seu tratamento; e afirmou que todos os brasileiros devem lutar pela garantia do acesso racional aos medicamentos ${ }^{2}$. O presidente do CNS também discordou da avaliação do ministro e afirmou que, aproximadamente, 1 milhão de pessoas que eram atendidas pelas unidades próprias do PFPB não teriam mais acesso direto e facilitado aos medicamentos antes ofertados. Criticou a mudança na política pública na área da saúde e afirmou:

É a sinalização de uma substituição de um entendimento da Constituição em que a saúde é vista como direito por uma lógica da saúde como mercadoria, em que você vai lá e compra no mercado, e quem regula esse processo são as leis do mercado. Acaba abalando a lógica de um sistema universal de saúde e vai na direção de um sistema que pensa em uma cobertura mínima, um sistema pobre para pobre ${ }^{\mathbf{2 ( 4 )}}$.

Outro posicionamento foi do Presidente do CRM do Estado de São Paulo, que afirmou ver com preocupação o fechamento das unidades próprias do PFPB por dificuldades dos pacientes na aquisição dos medicamentos ${ }^{2}$.

Novos critérios de acesso aos medicamentos no ATFP foram estabelecidos em 2018, e houve revisão dos valores repassados às farmácias credenciadas. O MS publicou uma notícia revelando que estudos internos demonstraram que o preço da Insulina no ATFP era 2,5 vezes mais caro do que na rede pública no $\mathrm{SUS}^{2}$, e informou o início de uma rodada de negociações entre a indústria farmacêutica e o setor varejista para debater o sobrepreço identificado. Esse processo foi ampliado, constatando-se diferenças de preços em outros itens do ATFP2.

Com o argumento de minimizar fraudes, o ministro afirmou que a revisão representaria economia de $\mathrm{R} \$ 750$ milhões, e o custo do programa passaria de $\mathrm{R} \$ 2,6$ bilhões para $\mathrm{R} \$ 1,85$ bilhão, atendendo o mesmo número de brasileiros. A redução de valores para as farmácias credenciadas provocou conflitos com o mercado farmacêutico ${ }^{2}$, e, em contraposição às afirmativas do ministro de que praticaria reajuste de preços no ATFP, a Associação Brasileira de Redes de Farmácias e Drogarias (Abrafarma) emitiu um comunicado público questionando o MS, afirmando que as reduções de preços deixariam seus custos descobertos'.

Em abril de 2018', entrou em vigor a Portaria MS $n^{0} 739$, atualizando os preços dos medicamentos do ATFP, com redução média de $38 \%$ do preço pago aos varejistas. Esse fato gerou pressão política do setor, sob alegação de que os preços definidos pelo MS inviabilizariam o programa, prejudicando o acesso 
aos medicamentos pela população. $\mathrm{O}$ presidente do Sindicato da Indústria de Produtos Farmacêuticos do Estado de São Paulo afirmou que a medida pode provocar um grande número de pedidos de descredenciamento de farmácias. Deve-se considerar que a decisão do ministro, ao reduzir a margem de lucro das farmácias credenciadas, pode desestimular a rede varejista pelo impacto no orçamento produzido com as vendas do programa. Ainda nesse momento, já houve descredenciamento, pelo MS, de, aproximadamente, $5 \%$ das farmácias do ATFP, com o argumento de irregularidades ou suspeita de fraudes na execução do programa'.

Tais fatos revelam a intensa disputa do mercado farmacêutico com o Estado brasileiro, seja indústria, seja rede varejista, mesmo porque o ATFP tem representado um forte impulsionador das vendas de medicamentos no Brasil. Numa conjuntura extremamente desfavorável aos direitos sociais e ao SUS, o conjunto de fatos dos últimos dois anos tem contribuído para a reflexão e a compreensão do PFPB quando se agudizaram conflitos de interesse em torno da questão MAF.

Restaria sinalizar que o ministro relator do processo que investiga o programa e questiona a eficácia do uso do dinheiro público nas parcerias com farmácias privadas, ao ser entrevistado, afirmou que a preocupação do TCU, como órgão fiscalizador, é avaliar se a decisão pelo encerramento do PFPB está devidamente fundamentada em estudos técnicos que comprovem ser essa a melhor solução do ponto de vista orçamentário e também da saúde pública'. A síntese dos principais fatos relacionados ao $\mathrm{PFPB}$ pode ser visualizada na figura 1 .

Figura 1. Linha do tempo do Programa Farmácia Popular do Brasil

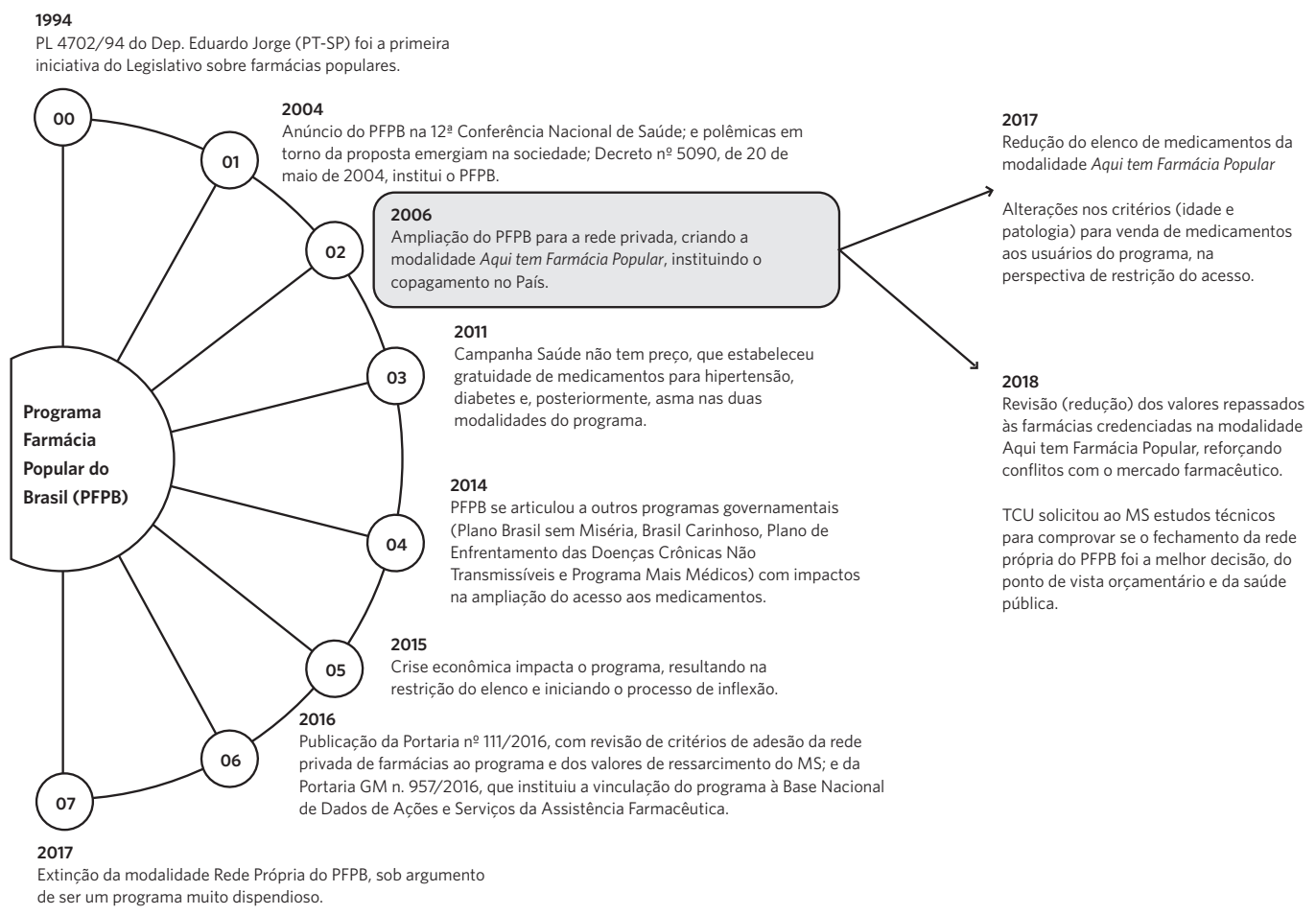




\section{Comentários finais}

A despeito das críticas sobre ambas as modalidades do PFPB e do rechaço inicial pela equipe responsável pela implantação da proposta, o programa resultou em ampliação do acesso a medicamentos essenciais, conforme constatado por estudos. Nesse aspecto, essa política farmacêutica se aproximou da pauta relativa à questão MAF defendida pelo movimento sanitário. Entretanto, permanecem lacunas concernentes à qualidade do acesso em suas diversas dimensões de análise e à promoção do uso racional de medicamentos, na perspectiva da integralidade da AF.

As políticas farmacêuticas estabelecidas com a PNM e a PNAF não propuseram a reorganização da AF tão somente na disponibilização de medicamentos. Isso pode explicar porque, entre farmacêuticos/atores da RSB, a criação do PFPB representasse uma contradição, dados os desafios que a AF na rede própria do SUS comportava, e ainda comporta: problemas de estrutura física inadequada; ausência de farmacêuticos nas farmácias; controle de estoque deficitário, entre outros. Em síntese, dificuldades relacionadas à insuficiente institucionalização dos serviços farmacêuticos no SUS ${ }^{32}$. Esses desafios não seriam superados com o PFPB, e a AF permanece com problemas.

Barros e colaboradores ${ }^{33}$ encontraram aspectos relacionados à institucionalização da AF fortemente associados com o acesso a medicamentos na atenção básica, demonstrada com os indicadores ferramentas de gestão, financiamento, estrutura de pessoal, participação e controle social. Concluem que os resultados indicam a necessidade de institucionalizar plenamente a AF para sua consolidação e efetividade dos serviços farmacêuticos públicos.

$\mathrm{O}$ processo de institucionalização da política de $\mathrm{AF}$ e, consequentemente, seus resultados, podem ser influenciados pelas concepções de AF que predominarem em determinado governo. Os múltiplos entendimentos de AF encontrados nos discursos de atores estratégicos traduzem não só os sentidos das práticas como expressam disputas de sentidos que existem na área e que envolvem o medicamento, um objeto complexo, produtor de sentidos, ao mesmo tempo, mercadoria, bem social, tecnologia em saúde e meio de trabalho. Reverter a lógica de se conceber a AF como ação mais procedimental e centrada no medicamento para outra centrada no cidadão é um processo gradual. Afinal, trata-se de um processo social, histórico, com subjetividades, visões de mundo, ideologias que transcendem os arranjos legais, logísticos e técnico-administrativos no sistema de saúde ${ }^{25}$.

A institucionalização da AF pressupõe o entendimento do seu caráter transversal e sistêmico, de modo a efetivar a internalização de procedimentos e rotinas nas organizações componentes dos diversos espaços operativos do SUS. Esse processo se realiza mediante o desenvolvimento de um conjunto de atividades de planejamento e gestão dos serviços farmacêuticos e de práticas que requerem competências técnicas e gerenciais para assegurar qualidade na seleção, programação, aquisição, dispensação dos medicamentos, e atividades de planejamento terapêutico de caráter clínico, visando ao uso racional dos medicamentos ${ }^{32}$, bem como a prevenção e a resolução de problemas farmacoterapêuticos experimentados pelos usuários, causadores de significativa morbimortalidade relacionada ao uso de medicamentos ${ }^{22}$.

A efetividade das políticas farmacêuticas requer a implementação das diretrizes da PNM e dos princípios da PNAF em sua inteireza e um conjunto de práticas, a exemplo de serviços farmacêuticos clínicos, de farmacovigilância, controle da propaganda de medicamentos, entre outros, que dificilmente encontrarão condições para serem realizadas nos estabelecimentos farmacêuticos do ATFP, cuja concepção de AF, desde sua origem, é restrita ao acesso a medicamentos. 
Compreende-se, assim, que a efetividade da AF como um direito social depende de sua integração nos serviços públicos de saúde, de modo a prover tanto o acesso aos medicamentos quanto às ações necessárias à promoção do uso racional, contrapondo-se à lógica do medicamento como mercadoria, cuja característica mais enfatizada é exatamente o custo. No Brasil, mantém-se um conjunto de desafios à integralidade da $\mathrm{AF}$, tal como aquele referente à preservação do SUS, com seus princípios e diretrizes.

\section{Referências}

1. Observatório de análise política em saúde. Eixo de Política de Medicamentos, de Assistência Farmacêutica e Vigilância Sanitária [internet]. Salvador: OAPS; c2018 [acesso em 2018 jul 5]. Disponível em: http://analisepoliticaemsaude.org/oaps/eixos/ producao-cientifica/bf9689622d73e9bb52de07b97d81a297/.

2. Observatório de análise política em saúde. Eixo de Política de Medicamentos, de Assistência Farmacêutica e Vigilância Sanitária [internet]. Salvador: OAPS; 2017 [acesso em 2018 mar 1]. Disponível em: http://analisepoliticaemsaude.org/oaps/eixos/producao-cientifica/ bf9689622d73e9bb52de07b97d81a297/.

3. Alencar TOS, Paim JS. Reforma Sanitária Brasileira e políticas farmacêuticas: uma análise dos fatos produzidos entre 2003 e 2014. Saúde debate. 2017 set; 41(esp.3):45-59.

\section{Colaboradores}

\begin{abstract}
Alencar TOS, Araújo OS, Costa EA, Barros RD e Lima YOR contribuíram para a concepção e o planejamento ou para a análise e a interpretação dos dados; Paim JS contribuiu para a revisão crítica do conteúdo e participou da aprovação da versão final do manuscrito.
\end{abstract}

4. Brasil. Ministério da Saúde Os 10 anos do Programa Farmácia popular do Brasil. In: VII Fórum Nacional de Assistência Farmacêutica. Brasília, DF: MS; 2014.

5. Costa EA, Araújo PS, Pereira MT, et al. Situação sanitária dos medicamentos na atenção básica no Sistema Único de Saúde. Rev Saúde Pública [internet]. 2017 [acesso em 2018 abr 4]; 51(supl):2-12. Disponível em: https://doi.org/10.11606/S015188787.2017051007106.

6. Instituto Brasileiro de Geografia e Estatística. Pesquisa Nacional de Saúde - 2013. Percepção do estado de saúde, estilos de vida e doenças crônicas: Brasil, grandes regiões e unidades da federação. Rio de Janeiro: IBGE; 2014 [acesso em 2015 nov 12]. Disponível em: ftp://ftp.ibge.gov.br/PNS/2013/pns2013. pdf. 
7. Silva RM, Caetano R. Programa "Farmácia Popular do Brasil” caracterização e evolução entre 20042012. Ciênc Saúde Coletiva. 2015 out; 20(10):29432956.

8. Miranda VIA, Fassa AG, Meucci RD, et al. Utilização do Programa Farmácia Popular do Brasil por idosos. Rev Saúde Pública. 2016; 50(13):1-13.

9. Pereira MA. Programa Farmácia Popular no Brasil: uma análise sobre sua relação com o Complexo Econômico-Industrial da Saúde e os programas estratégicos do Governo Federal [dissertação]. Rio de Janeiro: Fiocruz; 2013. 104 p.

10. Ferreira PAA. Efeitos do copagamento de medicamentos sobre saúde no Brasil: evidências do programa Aqui Tem Farmácia Popular. Rev BNDES. 2017 jun; 47:67-77.

11. Yamauti SM, Barberato-Filho S, Lopes LC. Elenco de medicamentos do Programa Farmácia Popular e a Política Nacional de Assistência Farmacêutica. Cad Saúde Pública. 2015 ago; 31(8):1648-1662.

12. Alencar TOS. A Reforma Sanitária Brasileira e a questão medicamentos/assistência farmacêutica [tese]. Salvador: UFBA; 2016. $439 \mathrm{f}$.

13. Bardin L. Análise de conteúdo. 7. ed. São Paulo: Edições 70; 2011.

14. Heller A. A teoria das necessidades em Marx. 2. ed. Barcelona: Ediciones Península; 1986.

15. Marx K. Introdução à crítica da Economia Política. In: Marx K. Os pensadores. São Paulo: Nova Cultural; 1996. p. 25-54.

16. Testa M. Pensamento estratégico e lógica de programação: o caso da saúde. São Paulo: Hucitec; Rio de Janeiro: Abrasco; 1995.

17. Testa M. Vida. Senãs de Identidad (Miradas al Espejo). Salud Colectiva. 2005; 1(1):33-58.

18. Pinto CBS. O Programa Farmácia Popular do Bra- sil: modelo, cobertura e utilização frente à Política Nacional de Medicamentos [dissertação]. Rio de Janeiro: Fiocruz; 2008. 153 p.

19. Carvalho G. Farmácia popular sim, pagamento por remédios não! Saúde debate. 2004 maio-ago; 28(67):173-175.

20. Burstall M.L. The management of the cost and utilization of pharmaceuticals in the United Kingdom. Health Policy. 1997 set; 41(supl):27- 43.

21. Fattore G, Jommi C. The new pharmaceutical policy in Italy. Health Policy. 1998; 46(1):21-41.

22. Araújo PS, Costa EA, Guerra Junior, AA et al. Atividades farmacêuticas de natureza clínica na atenção básica no Brasil. Rev Saúde Pública [internet]. 2017 [acesso em 2018 ago 26]; 51(suppl.2):1-11. Disponível em: http://www.scielo.br/pdf/rsp/v51s2/pt_00348910-rsp-S1518-51-s2-87872017051007109.pdf.

23. Cipolle RJ, Strand LM, Morley PC. O Exercício do Cuidado Farmacêutico. Brasília, DF: Conselho Federal de Farmácia; 2006.

24. Zubioli, A. Prefácio. In: Alencar TOS, Nascimento MAA, Alencar BR. Assistência farmacêutica no SUS: articulando sujeitos, saberes e práticas. Feira de Santana: UEFS; 2011. p. 9-15.

25. Costa EA, Araúli PS, Penaforte TR et al. Concepções de assistência farmacêutica na atenção primária à saúde, Brasil. Rev Saúde Pública [internet]. 2017 [acesso em 2018 jun 28]; 51(suppl.2):1-11. Disponível em: http://www.scielo.br/pdf/rsp/v51s2/pt_00348910-rsp-S1518-51-s2-87872017051007107.pdf.

26. World Health Organization. The Pursuit of Responsible Use of Medicines: Sharing and Learning from Country Experiences. Genebra: WHO; 2012 [acesso em 2016 jan 10]. Disponível em: http://apps.who. int/iris/bitstream/10665/75828/1/WHO_EMP_ MAR_2012.3_eng.pdf.

27. Scaramuzzo M. Genéricos avançam em farmácia popular. Valor Econômico [internet]. $2011 \mathrm{set}$ 
28 [acesso em 2011 out 28]. Disponível em: http:// www.pressreader.com/brazil/valor-econ\%C3\%B4 mico/20110928/281848640342634.

28. Menezes L. Genéricos: Fim de patentes contribui para elevar as vendas. Rev Valor Setorial Saúde [internet]. 2012 [acesso em 2018 jun 8]. Disponível em: http://www.abradilan.com.br.

29. Silva RM, Caetano R. Custos da assistência farmacêutica pública frente ao Programa Farmácia Popular. Rev Saúde Pública. 2016; 50(74):1-11.

30. Garcia MM, Acúrcio FA. Avaliação econômica dos programas Rede Farmácia de Minas do SUS versus Farmácia Popular do Brasil. Ciênc Saúde Colet. 2017; 22(1):221-233.
31. Vieira FS. Integrality of the therapeutic and pharmaceutical care: a necessary debate. Rev Saúde Pública. 2017 dez; 51(126):2-6.

32. Souza GS, Costa EA, Barros RD, et al. Caracterização da institucionalização da assistência farmacêutica na atenção básica no Brasil. Rev Saúde Pública. 2017; 51(supl.2):1-12.

33. Barros RD, Costa EA, Santos DB, et al. Acesso a medicamentos: relações com a institucionalização da assistência farmacêutica. Rev Saúde Pública. 2017; 51(supl.2):1-11.

Recebido em 14/07/2018

Aprovado em 27/09/2018

Conflito de interesses: inexistente

Suporte financeiro: Conselho Nacional de Desenvolvimento

Científico e Tecnológico (CNPq) e Ministério da Saúde (MS) 